\title{
Effect of discrimination on food decisions
}

\author{
Elizabeth A. Pascoe ${ }^{1}$ and Laura Smart Richman ${ }^{2}$ \\ ${ }^{1}$ School of Psychological Sciences, University of Northern Colorado, Greeley, CO, USA \\ ${ }^{2}$ Department of Psychology and Neuroscience, Duke University, Durham, NC, USA
}

This research examined effects of discrimination on food decisions. In Study 1, reflecting upon past experiences of discrimination, as compared to a neutral topic, caused an increased desire to consume unhealthy foods. In Study 2, participants received a negative evaluation from a biased or fair grader. Past experiences with discrimination moderated how people responded to the feedback. Those participants who had infrequent past experiences with discrimination were most likely to endorse unhealthy food options after receiving the biased evaluation. Those who scored high on past discrimination were unaffected by experimental condition and endorsed similar numbers of healthy and unhealthy food options after receiving the evaluative feedback. When offered an actual snack, those who accepted one were more likely to choose an unhealthy option following discrimination, regardless of past discrimination level. These results suggest that discrimination may be affecting self-regulatory capacity in regard to food choices.

Keywords: Discrimination; Health behaviors; Decision making; Self-regulation.

Research on the impact of discrimination on health has most often focused on the mental and physical stress effects that such experiences evoke, such as changes to individual's sense of self-worth and increased vulnerability to depression, anger, or anxiety (see Williams, Neighbors, \& Jackson, 2003, for review), elevated cardiovascular reactivity (see Brondolo, Rieppi, Kelly, \& Gerin, 2003) and decreased nighttime dipping, where blood pressure rates while sleeping change less than $10 \%$ from daytime levels (Richman, Pek, Pascoe, \& Bauer, 2010). Such disruptions from mental and physical homeostasis, if experienced frequently, may increase individuals' risk for a multitude of diseases (Nemeroff, 1996; Smith et al., 2005; Treiber et al., 2003; Walker \& Diforio, 1997).

Another pathway by which discrimination may exact a toll on health is through behavioral responses to these discrimination experiences. In a metaanalytic review of this topic, Pascoe and Smart Richman (2009) found that perceiving discrimination was highly correlated with decreased participation in healthy behaviors in domains of eating, exercising, resting, medication adherence and routine cancer and diabetic screening. Discrimination was also highly correlated with increased participation in unhealthy behaviors including alcohol use and abuse, smoking and tobacco use, and other drug abuse. The relationship between perceived discrimination and health behaviors suggests that such behavioral responses may be one of the mechanisms by which the experience of perceiving discrimination may negatively affect health outcomes. However, the

Correspondence should be addressed to: Elizabeth Pascoe, School of Psychological Sciences, University of Northern Colorado, Campus Box 94, Greeley, Colorado, 80639, USA.

E-mail: Elizabeth.pascoe@unco.edu 
evidence of this pathway has thus far been largely correlational, and the causal nature of this link is still unclear.

One explanation for how perceived discrimination may influence health-related behavior is that such experiences produce subtle lapses in self-control, which may unconsciously hinder an individual's subsequent ability to make appropriate health-related choices. Much research supports the existence of a single, domaingeneral resource of self-control from which individuals draw every time they exert self-control (Muraven, Tice, \& Baumeister, 1998). The capacity of this resource is limited in that each exertion of self-control can affect subsequent self-regulation. Everyday behaviors such as resisting temptations, delaying gratification, monitoring impressions of others, and controlling emotional reactions require self-control, contributing to the slow depletion of one's self-regulatory resource throughout the day.

The exclusion literature provides some evidence that the experience of being excluded or cast as an "outsider" is related to self-regulatory deficits. Exclusion has been found to predict an increased likelihood of participating in self-defeating behaviors such as taking foolish risks in money games and favoring procrastination over studying for an upcoming test (Twenge, Catanese, \& Baumeister, 2002). After being excluded, individuals have also been found to make poor health-related choices, such as opting for candy bars over granola bars or reading entertainment magazines instead of participating in a health-improvement survey (Twenge et al., 2002). Negative mood was not found to mediate these results, suggesting that emotional distress is not a necessary prerequisite for self-regulatory breakdown.

Recent work on stereotype threat suggests that attempts to suppress stereotypeconsistent behavior and negative emotion resulting from threatened identity can not only threaten individual performance within the stereotype-relevant domain but can also "spill over" and affect behavior in stereotype-irrelevant domains (Inzlicht \& Kang, 2010). In a series of studies, Inzlicht and Kang (2010) found that women who felt stereotype threat on a difficult math test behaved more aggressively toward partners who had previously evaluated them negatively, ate more ice cream in a taste-test, and were more likely to engage in a risky lottery than women who were instructed to view the math test objectively. These findings suggest that the processes involved in managing responses to such threat also consume self-control resources and subsequent efforts to exert self-control can become compromised.

These findings and recent theorizing on the commonalities of rejection-related experiences (Richman \& Leary, 2009) provide a foundation for predicting that when people experience discrimination, as with other forms of social exclusion, they exhibit subsequent declines in their capacity to self-regulate. This vulnerability could potentially affect individual health by making it harder to resist engagement in risky, yet tempting, activities such as unhealthy eating, drug use, alcohol use, or unprotected sex.

The current research examined a causal pathway of resource depletion between discrimination and food-related choices, thus identifying a potential mechanism by which discrimination may affect health outcomes. In Study 1, participants recalled a past experience of racial discrimination or a neutral experience and then indicated their food preferences on a forced-choice computer task. In Study 2, participants experienced negative feedback that was attributed to either discrimination or an unbiased evaluation and then engaged in the same food-choice task as in Study 1. They were also offered actual food that was either unhealthy or not. We also measured frequency of past experience of discrimination to explore whether the 
amount of exposure to discrimination could affect vulnerability to self-regulatory deficits.

\section{Study 1}

Previous research on the recollection of past personal experiences of discrimination and self-regulatory resource depletion has shown that individuals who wrote about discrimination showed decreased persistence at squeezing a hand grip when compared with individuals who wrote about their daily routine (Richman, 2010). The current study extended these results by asking individuals to make a variety of hypothetical food-related choices after reflection upon a single personal experience of discrimination. Individuals who reflected on a past experience of discrimination were expected to exhibit more negative food-related decisions than control individuals, suggesting that reflection upon discriminatory experiences may be more depleting than reflection upon everyday topics, subsequently contributing to more dysregulated health-related decision making.

\section{Method}

\section{Participants}

Participants were 69 African-American undergraduates $(71 \%$ female, age $M=20.04$ ). Excluded from the analyses were data from three individuals who were suspicious of the study's purpose before measurement of the outcome variables (total analysis $n=66)^{1}$

\section{Procedure}

Individuals were told they would be participating in a study examining how writing tasks are related to performance in everyday decision making. Individuals completed the study at personal computers in private rooms.

Discrimination manipulation. Participants were randomly assigned to reflect upon one of two topics for three minutes. Half of participants $(N=33)$ were asked to mentally reflect on a time when they were treated unfairly due their race or ethnicity and half $(N=33)$ were asked to reflect upon their daily routine. After three minutes of reflection, all participants were instructed to type for 10 minutes about the experience.

Food-decision task. After the writing task, participants were presented with pictures of either two food items or two beverage items shown side by side on the computer screen and were instructed to choose, by pressing a button on the screen below the picture of their choice, which food or beverage option they would be most likely to eat or drink if both options were presented to them at that very moment. Participants were instructed to make their decision as quickly as possible.

The trials were divided such that in 20 trials, two healthy options were presented simultaneously, 20 trials paired two unhealthy options, and, in the remaining 40 trials (target trials), one food or beverage option presented was healthier than the other (e.g., fried chicken sandwich vs. grilled chicken sandwich). Trial type was randomized between participants. Participants' choices on trials that paired unhealthy and healthy foods together were scored as "1" when the unhealthy 
option was chosen and " 0 " when the healthier option was chosen. Target trial scores were summed to create an index of predicted eating behavior.

Following participation in the food-decision task, individuals recorded their gender, age, year in school, and family income level. These demographic variables, along with individuals' self-reported importance of healthy eating have been found to affect participation in healthy behaviors, and thus were included as covariates in all analyses (e.g., Iyer \& Haslam, 2003; Martin, Tuch, \& Roman, 2003; Whitbeck, 2001). Self-reported past discrimination, which may influence individual perception and response to experiences of negatively valenced events (Richman, Bennett, Pek, Siegler, \& Williams, 2007) was also measured.

Past discrimination. A 10-item version of the everyday discrimination scale measuring the frequency of experiencing a variety of forms of everyday interpersonal mistreatment over the previous 12 months (Williams, Yu, Jackson, \& Anderson, 1997) was included in order to determine whether past exposure to discrimination moderated the effects of recalling such an experience An additional item was included, "People ignore you or act as if you are not there" based on Matthews, Salomon, Kenyon, and Zhou (2005). The overall reliability was high $(\alpha=.83)$. The items were scored along a 4-point scale with higher scores reflecting higher levels of past discrimination.

Eating habits. The importance that individuals place on healthy eating was assessed through nine questions from the Diet and Health Knowledge Survey Short Form (United States Department of Agriculture, 1996; York-Crowe, White, Paeratakul, \& Williamson, 2006), since such knowledge and past behavior could influence current food choices. Questions were summed to create an index of the importance of healthy eating for each individual.

After completing these measures, participants were probed for suspiciousness. Participants were asked about their thoughts on the study, including their presumed purpose of the experiment. Participants were also asked to explain their particular food endorsements. In particular, participants were asked whether they believed they would normally make the same food-related decisions they did during the experiment. If participants indicated their experimental choices were different from their everyday choices, they were asked to specify the reason they made an unusual choice during the experiment.

\section{Results and Discussion}

Regression analysis was used to examine whether discrimination condition affected participants' total number of unhealthy target food decisions, controlling for age, gender, year in school, family income, the importance an individual placed on consuming a healthy diet, along with the potential moderator of past discrimination. Variables were mean centered before inclusion in the regression equation.

Results indicated a significant main effect of discrimination condition, $B=0.258$, $t(58)=2.521, p<.02 .^{2}$ Individuals who reflected upon an experience of racial discrimination chose more unhealthy foods on target trials of the food decision task $(M=21.06)^{3}$ than individuals in the control condition $(M=18.24)$. Investigation of a potential moderating effect of past discrimination revealed no significant effect $(p>.05)$ and the addition of the interaction term did not alter the effect of discrimination condition on unhealthy food decisions. 
These results suggest that reflection upon an experience of discrimination may be depleting and may make subsequent self-regulatory efforts more difficult. However, would this suggest that individuals who thought about a discriminatory experience might make actual unhealthy food choices following this reflection? Although past research has used hypothetical tasks to test self-regulatory failure (Galliot \& Baumeister, 2007; Finkel \& Campbell, 2001), this study was limited in that it did not require participants to make a real-life food choice akin to the either/or computer food-decision task, and thus was only hypothetical in determining individuals' food preferences following reflection upon discrimination. Requiring individuals to make an actual food decision would provide stronger evidence that discrimination experiences may influence individuals' real-world decisions.

Furthermore, this study cannot specify whether the effects were due to reflection upon a discriminatory event or upon a negative event in general. It is possible that if individuals in the control condition had reflected upon negative events such as death, illness, or pain, no differences would have been found between experimental conditions. Study 2 was designed to address these limitations.

\section{Study 2}

In study 2, we examined food preferences and behavior in response to another form of discrimination: gender bias. In this study, we predicted that negative feedback from a biased reviewer (an in-the-moment experience of discrimination) could result in less healthy food preferences as compared to negative feedback from a fair reviewer. This control group allowed for the potential to better distinguish effects of discrimination from other negative evaluative experiences. Individuals who received negative feedback from a biased grader were expected to show less healthy food decisions on both hypothetical and actual food-choice tasks than individuals who received negative feedback from a fair grader.

\section{Method}

\section{Participants}

Participants were 109 female undergraduates (48.9\% Caucasian, 20.2\% Asian, $20.2 \%$ Black, age $M=19.91)$. Excluded from the analyses were data from 14 individuals who were suspicious of the study's evaluator before measurement of the outcome variables (total analysis $n=95){ }^{4}$

\section{Procedure}

Participants were told this study examined the relationship between creativity and performance in everyday activities and decision making, and that their own level of creativity ability would be evaluated by a judge. Individuals were seated alone in a quiet room and were asked to answer several questions purportedly to assess creativity level and problem-solving techniques (Inman, 2001). After giving participants 10 minutes to answer the questions, the researcher collected participant answers and ostensibly took them to be graded while the participant read an assigned article.

Discrimination manipulation. Participants were randomly assigned to receive one of two comments about the evaluator, delivered right before "grading" occurred. Approximately half of participants $(N=44)$ were told that the grader had 
consistently rated women's answers as less creative than men's answers when he did similar grading for a different study (discrimination condition). The remaining participants $(N=53)$ were told that the grader was fair, and that his ratings of answers were representative of individuals' actual creativity levels (control condition).

The researcher returned five minutes later, telling all participants that they received a $71 \%$, or a "C". Participants were left alone in the room for a minute while the researcher "prepared" the next task. This time was meant to give participants a chance to briefly process the negative feedback before completing the target outcome measures.

Food-decision task. Upon returning, the researcher instructed participants to sit in front of a computer in another room, where participants engaged in the same food-decision task described in Study 1.

Candy bar or granola bar choice. When participants reached the end of the fooddecision task, they were instructed to notify the researcher, who re-entered the room carrying a basket containing a variety of candy and healthy "all-natural" granola bars. Participants were allowed to select any treat as a "thank you" for participation while the researcher queued up the next survey. Researchers recorded participants' snack choices after leaving the room. Participants then completed the same battery of computer questionnaires including demographic information, past discrimination and diet information as described in Study 1 and the additional covariate of race.

Attributions to discrimination. Finally, individuals were asked a series of three questions to assess their attributions for their creativity scores. Individuals were asked on a scale from 0 (not at all) to 10 (a great deal) how much they believed their creativity score was due to: (a) their actual creative ability; (b) the effort they put into the task; and (c) discrimination on the part of the grader. Higher scores indicated greater attributions to the reason mentioned. These attributions were used as a check of the discrimination manipulation.

After completing these measures, participants were probed for suspiciousness. Anyone who indicated that they didn't think there was an actual grader or that their work was not evaluated by someone else were not included in the analyses $(N=14)$.

\section{Results and Discussion}

Individuals' attributions for their creativity score were examined by experimental condition as a manipulation check. Participants in the discrimination condition were more likely to attribute their negative score to discrimination on the part of the grader $(M=3.80)$ than those in the non-biased condition $(M=1.21), t(94)=-8.71$, $p<.01$. Furthermore, those in the discrimination condition were not more likely to attribute their negative score to ability $(M=7.25)$ or effort $(M=6.81)$ than participants in the non-biased condition (ability $M=7.25$, effort $M=7.57, p s>.05$ ).

As in Study 1, regression analysis was used to examine the effect of discrimination condition on the total number of unhealthy decisions on target trials of the computer task while controlling for age, race, year in school, income, importance of a healthy diet, and including past discrimination as a moderator. Results indicated no main effect of experimental condition or past discrimination on the food-decision task ( $p s>.05)$, however, amount of past year discrimination was found to interact 
significantly with experimental condition, $B=-0.095, t(86)=-2.41, \quad p<.02$. Simple slopes analyses were performed to investigate the moderating effect of past discrimination. Predicted values for the interaction were calculated at one standard deviation above and below the means of the relevant independent variables (Aiken \& West, 1991). Simple slopes analyses revealed that individuals one standard deviation below the mean on past discrimination chose significantly more unhealthy options after receiving feedback from a biased reviewer $(M=15.91)$ than from a non-biased reviewer $(M=12.09), B=4.04(1.84), t(87)=2.20, p<.04$ (see Figure 1). Experimental condition had no significant effect for individuals one standard deviation above the mean on past discrimination, fair reviewer: $M=16.25$, biased reviewer: $M=14.05, B=-1.75(1.77), t(87)=-0.99, p>.05$. Regardless of experimental condition, these individuals endorsed similar numbers of unhealthy foods.

Binary logistic regression analysis was used to examine whether the experience of discrimination affected real-world food decisions (snack choice) using the same covariates as before. Because many individuals refrained from accepting a snack altogether, snack choice was investigated by examining the type of snack chosen by individuals who accepted a snack from the experimenter $(N=74){ }^{5}$ Selection of a granola bar (control $N=29$; discrimination $N=16$ ) was coded as 0 and selection of a candy bar (control $N=13$; discrimination $N=16$ ) was coded as 1 . Experimental condition was found to have a significant effect on actual food choice $(B=1.13$, Wald $\chi^{2}=3.92, p<.05$, odd ratio: $3.10,95 \%$ CI: 1.01-9.47). Individuals in the discrimination condition were three times more likely to choose a candy bar over a granola bar than individuals in the control condition. Analysis exploring past discrimination as a potential moderator of experimental condition was not significant $(p>.05)$.

In sum, these results suggest that experiencing discrimination may increase the tendency for subsequent unhealthy food choices for individuals who do not often encounter discrimination as compared to a negative evaluation control group. For these individuals, dealing with discrimination may be more depleting than a similar, negative non-discriminatory event, reducing individuals' ability to exert self-control in subsequent food-related decisions.

Investigation of actual snack choice also supported the hypothesis that experiences of discrimination may affect individuals' regulation of actual food-related decisions. Many individuals in the real-life food decision were able to resist the snack, regardless of experimental condition. However, this resistance, while possibly due to greater selfregulatory control, could have also stemmed from a variety of sources such as differential hunger status, knowledge of allergic conditions, and dislike of the snacks presented. A focus on only the participants who chose a snack suggests that the experience of discrimination may increase one's likelihood of subsequently making poor food-related decisions (in choosing an unhealthy snack over a healthy snack), replicating the findings from Study 1 . This reveals a potential real-world effect on food decisions for individuals experiencing discrimination.

\section{General Discussion}

Previous studies have provided compelling evidence that perceived discrimination may be harmful to both physical and mental health. The present research experimentally tested the potential pathway between perceived discrimination and participation in healthy food-related behaviors from a self-regulatory perspective. Results from two studies show that the experience of discrimination may affect 


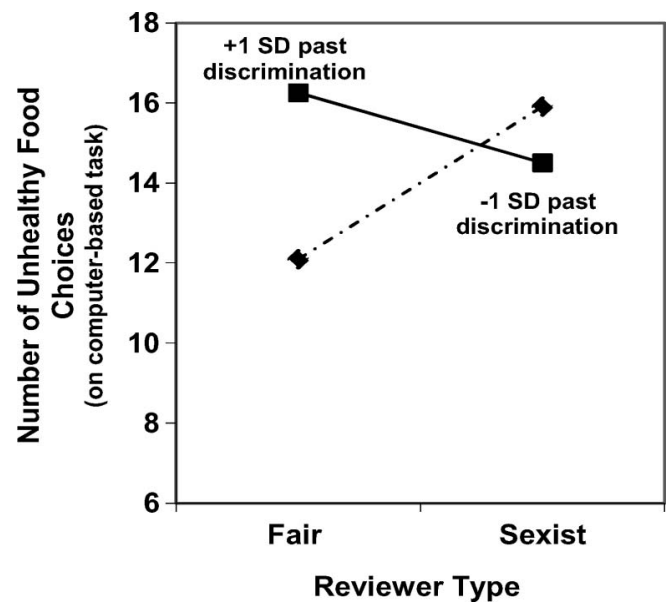

FIGURE 1 Number of unhealthy food choices selected on target trials for individuals one standard deviation above and below the mean in past year discrimination by experimental condition.

individuals' engagement in healthy food-related decision making. The cumulative effect of exposure to discrimination on caloric consumption is yet unknown, although longitudinal studies have found that increases in reports of everyday and lifetime discrimination are associated with increased consumption of fast food and weight gain (Cozier, Wise, Palmer, \& Rosenberg, 2009), suggesting potential longterm self-regulatory deficits in response to discrimination.

In our studies, past discrimination moderated responses to the experimental discrimination only in Study 2, in which female participants experienced gender discrimination. Those with low levels of past discrimination were more likely to make unhealthy food choices on the computer task after experiencing gender discrimination as compared to the negative feedback condition. Furthermore, past discrimination was not found to moderate responses to gender discrimination when choosing an actual real-world snack. Altogether, these differential results regarding the moderating effect of past discrimination suggest that certain features of an experience of discrimination such as group status may influence self-regulation more than others. The nature of the subsequent self-regulatory domain may also impact the likelihood of self-regulatory failure following discrimination. Many of the boundary conditions by which past exposure to discrimination may influence how people experience and respond to current exposure to discrimination are yet unknown, warranting further investigation.

The role of negative emotion in response to discrimination and self-defeating behaviors is an important area for future research. Past research has consistently linked the experience of negative emotion with participation in self-defeating behaviors (e.g., Baumeister \& Scher, 1988; Leith \& Baumeister, 1996). Recent work on discrimination and drug use has found that discrimination was associated with more drug willingness and use and that relationship was mediated by anger (Gibbons et al., in press). Because experiences of discrimination may be accompanied by heightened negative affect, it is possible this resulting emotional distress may mediate the link between discrimination and food choice. Although 
Study 2 attempted to equalize the negative affect felt between conditions by awarding negative feedback to both experimental conditions, it is possible that negative feedback from a sexist reviewer may have provoked more emotional distress than did receiving feedback from a fair reviewer. Research on the link between social exclusion and participation in self-defeating behavior and on self-regulation have found that breakdowns in self-regulation can occur independent of, or in the absence of, negative affect (Baumeister, Bratslavsky, Muraven, \& Tice, 1998; Twenge et al., 2002). Thus, emotional distress is not a necessary prerequisite for self-regulatory breakdown. However, future research on the causal pathway between discrimination and health decision making should investigate the potential mediating effects of emotional distress.

We interpret our findings within the context of a self-regulatory framework, but an alternative explanation may be that people prefer high-caloric foods after discrimination as a coping mechanism to relieve the negative emotions that were evoked. Discrimination makes them feel bad and they want to eat foods that may temporarily make them feel better. Additional work is needed to determine the strength of evidence for this interpretation, but our data provide some insight. Participants in both studies were asked, prior to debriefing, whether they believed they would normally make the same food-related decisions they did during the experiment. If participants believed their experimental choices may be different from their everyday choices, they were asked to specify the reason why they made different choices during the experiment. No participants indicated that their food choices were affected by the situation just experienced or that those foods were chosen to help them cope with the situation at hand. This suggests that such dysregulated eating may be reflective of nonconscious processes that may be more difficult to influence.

\section{Conclusion}

This research took a first step toward identifying the causal relationship between discrimination and health-related behaviors. Following two different manipulations of discrimination - recalling a past experience and receiving a biased evaluationparticipants exhibited preferences for more unhealthy foods. These findings provide initial evidence that experiences with discrimination may predict a reduced capacity to regulate behavior and may increase vulnerability to unhealthy food choices.

\section{Notes}

1. Examination of the outcome means for suspicious and non-suspicious participants across the two studies presented no consistent pattern of differences.

2. Since health-food knowledge could influence choices, we examined its effect in all analyses and found that results did not change with the inclusion of this measure.

3. Adjusted means are reported throughout this paper.

4. Individuals in the discrimination condition were more likely to report suspicion of the manipulation $(n=10)$ than individuals in the control condition $(n=4)$. Investigation of potential demographic differences across suspicious and non-suspicious individuals reveals that suspicious individuals were more likely to be upper-class men than nonsuspicious individuals, $t(106)=-2.04, p<.03$, and suspicious individuals tended to report higher levels of past discrimination than non-suspicious individuals, $t(106)=-2.14, p<.05$.

5. Two further regression analyses of snack choice used a three-category variable that included individuals who refrained from choosing a snack as the healthiest group 
(coded as -1 ; control $N=9$; discrimination $N=11$ ), and a dichotomous variable that grouped no choice and granola bar choice together as healthy options. In these analyses, experimental condition was found to have no significant effect on snack choice $(p s>.05)$. Analyses exploring the potential moderating effect of past discrimination were not significant $(p s>.05)$.

\section{References}

Aiken, L. S., \& West, S. G. (1991). Multiple regression: Testing and interpreting interactions. Newbury Park, CA: Sage.

Baumeister, R. F., Bratslavsky, E., Muraven, M., \& Tice, D. M. (1998). Ego depletion: Is the active self a limited resource? Journal of Personality and Social Psychology, 74, 12521265.

Baumeister, R. F., \& Scher, S. J. (1988). Self-defeating behavior patterns among normal individuals: Review and analysis of common self-destructive tendencies. Psychological Bulletin, 104, 3-22.

Brondolo, E., Rieppi, R., Kelly, K. P., \& Gerin, W. (2003). Perceived racism and blood pressure: A review of the literature and conceptual and methodological critique. Annals of Behavioral Medicine, 25(1), 55-65.

Cozier, Y. C., Wise, L. A., Palmer, J. R., \& Rosenberg, L. (2009). Perceived racism in relation to weight change in the Black Women's Health Study. Annals of Epidemiology, 19, 379387.

Finkel, E. J., \& Campbell, W. K. (2001). Self-control and accommodation in close relationships: An interdependence analysis. Journal of Personality \& Social Psychology, $81,263-277$.

Galliot, M. T., \& Baumeister, R. F. (2007). Self-regulation and sexual restraint: Dispositionally and temporarily poor self-regulatory abilities contribute to failures at restraining sexual behavior. Personality \& Social Psychology Bulletin, 33, 173-186.

Gibbons, F. X., Etcheverry, P. E., Stock, M. L., Gerrard, M., Weng, C.-Y., Kiviniemi, M., \& O'Hara, R. E. (in press). Exploring the link between racial discrimination and substance use: What mediates? What buffer? Journal of Personality \& Social Psychology.

Inman, M. L. (2001). Do you see what I see? Similarities and differences in victims' and observers' perceptions of discrimination. Social Cognition, 19(5), 521-546.

Inzlicht, M., \& Kang, S. K. (2010). Stereotype threat spillover: How coping with threats to social identity affects aggression, eating, decision making, and attention. Journal of Personality and Social Psychology, 99(3), 467-481.

Iyer, D. S., \& Haslam, N. (2003). Body image and eating disturbance among South Asian American women: The role of racial teasing. Eating Disorders, 34, 142-147.

Leith, K. P., \& Baumeister, R. F. (1996). Why do bad moods increase self-defeating behavior? Emotion, risk taking, and self-regulation. Journal of Personality and Social Psychology, 71(6), 1250-1267.

Martin, J. K., Tuch, S. A., \& Roman, P. M. (2003). Problem drinking patterns among African Americans: The impacts of reports of discrimination, perceptions of prejudice, and "risky" coping strategies. Journal of Health and Social Behavior, 44(3), 408-425.

Matthews, K. A., Salomon, K., Kenyon, K., \& Zhou, F. (2005). Unfair treatment, discrimination, and ambulatory blood pressure in Black and White adolescents. Health Psychology, 24, 258-265.

Muraven, M. D., Tice, M., \& Baumeister, R. F. (1998). Self-control as limited resource: Regulatory depletion patterns. Journal of Personality \& Social Psychology, 74, 774-789.

Nemeroff, C. B. (1996). The corticotrophin-releasing factor hypothesis of depression: New findings and new directions. Molecular Psychiatry, 1, 336-342.

Pascoe, E. A., \& Smart Richman, L. (2009). Perceived discrimination and health: A metaanalytic review. Psychological Bulletin, 135(4), 531-554. 
Richman, L. S. (2010). The effects of discrimination on self-regulatory capacity. Unpublished data, Duke University, Durham, NC.

Richman, L. S., Bennett, G., Pek, J., Siegler, I. C., \& Williams, R. B. (2007). Discrimination, dispositions, and cardiovascular responses to stress. Health Psychology, 26, 675-683.

Richman, L. S., \& Leary, M. (2009). Reactions to discrimination, stigmatization, ostracism, and other forms of interpersonal rejection: A multimotive model. Psychological Review, 116, 365-383.

Richman, L. S., Pek, J., Pascoe, E. A., \& Bauer, D. (2010). The effects of perceived discrimination on ambulatory blood pressure and affective responses to interpersonal stress modeled over 24-hours. Health Psychology, 29(4), 403-411.

Smith, G. D., Ben Shlomo, Y., Beswick, A., Yarnell, J., Lightman, S., \& Elwood, P. (2005). Cortisol, testosterone, and coronary heart disease: Prospective evidence from the Caerphilly Study. Circulation, 112, 332-340.

Treiber, F. A., Kamarck, T., Schneiderman, N., Sheffield, D., Kapuku, G., \& Taylor, T. (2003). Cardiovascular reactivity and development of preclinical and clinical disease states. Psychosomatic Medicine, 65, 45-62.

Twenge, J. M., Catanese, K. R., \& Baumeister, R. F. (2002). Social exclusion causes selfdefeating behavior. Journal of Personality \& Social Psychology, 83, 606-615.

United States Department of Agriculture. (1996). Diet and Health Knowledge Survey. From 1994-1996 Continuing Survey of Food Intakes by Individuals. http://www.ars.usda.gov/ SP2UserFiles/Place/12355000/pdf/dhks.pdf

Walker, E. F., \& Diforio, D. (1997). Schizophrenia: A neural diathesis-stress model. Psychological Review, 104, 667-685.

Whitbeck, L. B. (2001). Perceived discrimination and early substance abuse among African Indian children. Journal of Health and Social Behavior, 42(4), 405-424.

Williams, D. R., Neighbors, H. W., \& Jackson, J. S. (2003). Racial/ethnic discrimination and health: Findings from community studies. American Journal of Public Health, 93(2), 200208.

Williams, D. R., Yu, Y., Jackson, J. S., \& Anderson, N. B. (1997). Racial differences in mental health: Socio-economic status, stress and discrimination. Journal of Health Psychology, 2(3), 335-351.

York-Crowe, E. E., White, M. A., Paeratakul, S., \& Williamson, D. A. (2006). The Diet and Health Knowledge Survey: Development of a short interview format. Eating Behaviors, 7, 235-242. 
Copyright of Self \& Identity is the property of Psychology Press (UK) and its content may not be copied or emailed to multiple sites or posted to a listserv without the copyright holder's express written permission. However, users may print, download, or email articles for individual use. 